\title{
Design and Analysis of Shot Blasting Machine Hanger
}

\author{
Prof. Shital Bhosale ${ }^{1}$, Akshay Shelke ${ }^{2}$, Ayaj Shaikh ${ }^{3}$, Rohit Chavan ${ }^{4}$, Kishor Savant ${ }^{5}$ \\ (Assistant Professor Mechanical Engineering Department, J.S.P.M. (NTC) SPPU, Pune) 1 , \\ (Mechanical Engineering, J.S.P.M. (NTC) SPPU, Pune) 2,3,4,5 \\ Email:sbhosale17@gmail.com ${ }^{1}$,akshayshelke13@gmail.com²,ayajshaikh44@gmail.com ${ }^{3}$, \\ rchavan1997@gmail.com ${ }^{4}$,kishorshavant730@gmail.com ${ }^{5}$
}

\begin{abstract}
In Shot Blasting machine various hangers are used during shot blasting processes as per component size \& Types, in this work we worked out to increase production rate in current industry. For this it is required to increase hanger capacity for the various castings produced in the current industry. The shot blasting machine in foundry is $300 \mathrm{~kg}$. The current hanger capacity of shot blasting machine is 16 castings. Thus to increase hanger capacity of castings its required to modify the hanger such a way that its capacity is maximum up to $300 \mathrm{Kg}$ of short blasting machine. With the study of shot blasting machine and its hanger we should have to design new hanger as per capacity of machine. This work contains modification of current hanger, design of modified hanger, stress analysis by analytically and by using Ansys Software. Also in this work efficient and reliable design of hanger is find out.
\end{abstract}

Keywords - Shot blasting machine, hanger, casting (ape brake drum), and abrasive particles.

\section{INTRODUCTION}

Shot blasting is a surface finishing technique that involves rapidly impacting the surface of an object with a controlled stream of abrasive shot material. It is faster and more effective than filing for removing flash that may remain on a part after a casting or stamping process. It is also used for removing burrs, scale and rust that may interfere with the parts integrity, appearance or definition. Shot Blasting can also prepare a surface of a part for coating by removing surface contaminants and provide a surface profile for increase coating adhesion. Shot Blasting is different than shot peening which is used to induce compressive stresses on a parts surface for increased fatigue life, increase the part strength or preventing fretting. It uses different hangers to mount the various castings that requires to goes to process. The castings are mounted manually or by using automation for heavy castings purpose on the hanger. The different abrasives are used as per the requirements of different size and shape of abrasives.

\subsection{Existing Hanger Dimension}

$\begin{array}{lc}\text { Total Height } & :-700 \mathrm{~mm} \\ \text { Rod Diameter } & :-18 \mathrm{~mm} \\ \text { Hanger Hook Outer Día } & :-122 \mathrm{~mm} \\ \text { Total Horizontal length } & :-600 \mathrm{~mm} \\ \text { Middle Distance between } & \\ \text { two horizontal rod } & :-345 \mathrm{~mm}\end{array}$

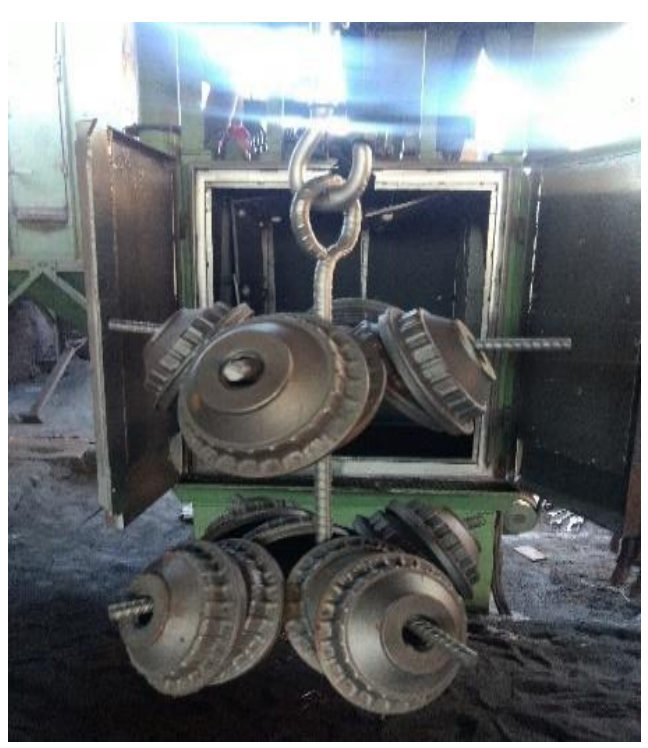

Fig.1. Existing Hanger with casting

\section{DESIGN OF NEW HANGER HEADINGS}

\subsection{Material of New hanger.}

New hanger made of mild steel having following properties,

$\begin{array}{lll}\text { New hanger material } & :- & \text { Steel }(\mathrm{Fe} 500) \\ \text { Ultimate tensile strength } & :- & 500 \mathrm{~N} / \mathrm{mm}^{2} \\ \text { Yield Strength } & :- & 290 \mathrm{~N} / \mathrm{mm}^{2}\end{array}$

2.2. Calculation for Diameter of steel rod.
Weight of Casting
Casting on Hanger
$:-4.5 \mathrm{Kg}$.
Casumg on Hanger 48 nos
Number of casting per branch: - 01 no 


\section{Available online at www.ijrat.org}
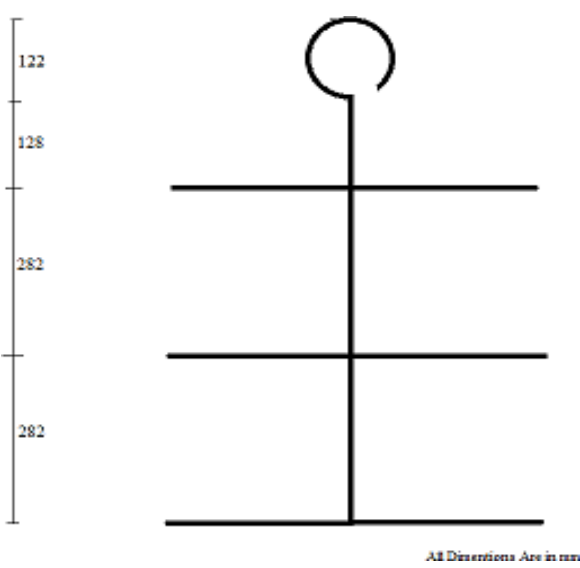

Fig. 2. Dimensions of new Hanger

\subsection{Load of castings acting per branch}

Load of single Casting $=4.5 * 9.81=44.145 \mathrm{~N}$

Considering $45 \mathrm{~N}$ for Calculation

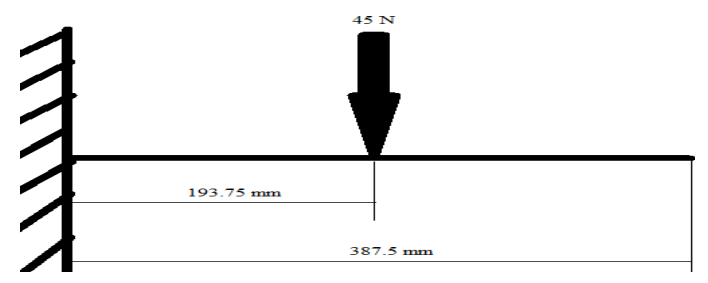

Fig. 3. Hanger branch as a cantilever beam

Bending Moment:-

$\mathrm{Mb}=\left(45^{*} 193.75\right)$

$\mathrm{Mb}=8718.75 \mathrm{~N}-\mathrm{mm}$

FOS: -1.5

Syt :- $290 \mathrm{~N} / \mathrm{mm}^{2}$

Working Stress $=$ Syt $/$ FOS

$=290 / 1.5=193.33 \mathrm{~N} / \mathrm{mm} 2$

Bending Stress $(\sigma \mathrm{b})$

$\sigma \mathrm{b}=32 \mathrm{Mb} / \pi \mathrm{d} 3$

$193.33=(32 * 8718.75) / \pi \mathrm{d} 3$

$\mathrm{d}=7.71 \mathrm{~mm} \mathrm{~d}=12 \mathrm{~mm}$

In Shot blasting machine, shots of abrasives hitting on the casting as well as hanger also due to this hanger wears, therefore Additional increased diameter is use because of this increase life of hanger.

Actual Bending Stress on hanger

$\sigma \mathrm{b}=32 \mathrm{Mb} / \pi \mathrm{d} 3$

$=(32 * 8718.75) / 123$

$\sigma \mathrm{b}=161.458 \mathrm{~N} / \mathrm{mm} 2$

Working stress $>$ Actual working stress

$193.33 \mathrm{~N} / \mathrm{mm} 2>161.458 \mathrm{~N} / \mathrm{mm} 2$

Hence design is safe

\subsection{Calculation of Middle RodDiameter}

Ultimate tensile strength $=500 \mathrm{~N} / \mathrm{mm}^{2}$

Factor of Safety $=1.5$

Working Stress $=$ Sut $/$ FOS

$500 / 1.5=333.33 \mathrm{~N} / \mathrm{mm} 2$

Stresses acting on middle Rod

i.e. Direct Stress and Bending Stress.

\section{Load on Single Casting $=45 \mathrm{~N}$}

Total load of Casting on Hanger $=45^{*} 48=2160 \mathrm{~N}$

Direct Stress $(\sigma d)=F / A=(2160 * 4) / \pi \mathrm{d} 2=2750.19 / \mathrm{d} 2$

Bending Moment on Single Branch $=8718.75 \mathrm{~N}-\mathrm{mm}$ Total Bending Moment $=8718.7 * 48=418500 \mathrm{~N}-\mathrm{mm}$

Bending Stress $(\sigma b)=(32 * \mathrm{Mb}) / \pi \mathrm{d} 3$

$=(32 * 418500) / \pi \mathrm{d} 3=4262805.996 / \mathrm{d} 3$

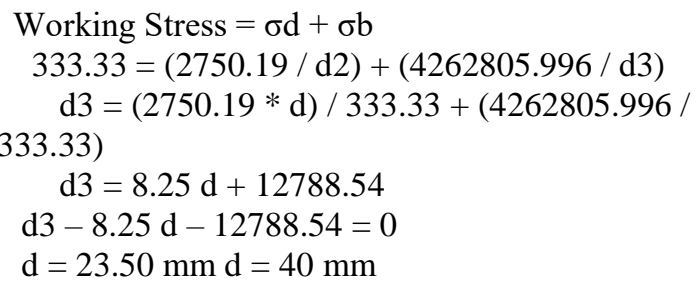

In Shot blasting machine, shots of abrasives hitting on the casting as well as hanger also due to this hanger wears, therefore Additional increased diameter is use because of this increase life of hanger.

Direct Stress $(\sigma \mathrm{d})=\mathrm{F} / \mathrm{A}=(2160 * 4) / \pi 402=1.71 \mathrm{~N} / \mathrm{mm} 2$

Bending Stress $(\sigma b)=(32 * \mathrm{Mb}) / \pi \mathrm{d} 3$

$$
\begin{aligned}
& =(32 * 418500) / \pi 403 \\
& =66.60 \mathrm{~N} / \mathrm{mm} 2
\end{aligned}
$$

Working Stress $=\sigma \mathrm{d}+\sigma \mathrm{b}$

$=1.71+66.60$

$=68.31 \mathrm{~N} / \mathrm{mm} 2$

Working stress $>$ Actual working stress

$333.33 \mathrm{~N} / \mathrm{mm} 2>68.31 \mathrm{~N} / \mathrm{mm} 2$

Hence design is safe

\subsection{Weight of Existing Hanger}

Weight of single branch

$\mathrm{W}=$ Volume $*$ Density

Where,

Density $=7850 \mathrm{Kg} / \mathrm{m} 3$

Radius $(\mathrm{r})=6 \mathrm{~mm}=0.006 \mathrm{~m}$

Length $(1)=387.5 \mathrm{~mm}=0.3875 \mathrm{~m}$

$\mathrm{W}=\pi \mathrm{r} 21 * 7850=\pi * 0.0062 * 0.3875 * 7850$

$$
=0.3440 \mathrm{Kg}
$$

Total Weight of Branches $=48 * 0.3440=16.51 \mathrm{Kg}$

Weight of Vertical Bar Radius (r) $=20 \mathrm{~m}=0.020 \mathrm{~m}$

Length $(1)=692 \mathrm{~mm}=0.692 \mathrm{~m}$ 
Available online at www.ijrat.org

Length of Hook $=\pi * d=3.14 * 0.122=0.3832 \mathrm{~m}$

Total Length of Hanger $=0.692+0.3832=1.075 \mathrm{~m}$ $\mathrm{W}=$ Volume $*$ Density

$$
\mathrm{W}=\pi \mathrm{r} 21 * 7850
$$

Weight $(\mathrm{W})=3.14 * 0.0202 * 1.075 * 7850=10.60 \mathrm{Kg}$

\subsection{Weight of circular ring}

Length of circular ring $=\pi * d=3.14 * 0.800$

$$
=2.51 \mathrm{~m}
$$

$\mathrm{W}=$ Volume $*$ Density

$\mathrm{W}=\pi \mathrm{r}^{2} 1 * 7850$

Weight $(\mathrm{W})=3.14 * 0.006^{2} * 2.51 * 7850=2.22 \mathrm{Kg}$ Total weight of circular ring $=2.22 * 3=6.66$ $\mathrm{kg}$ Total Weight of Hanger $=16.51+10.60+$ $6.66=33.77 \mathrm{Kg}$

Thus weight carrying capacity of shot blasting machine i.e. $300 \mathrm{~kg}$ therefore total weight with 48 no. of castings and hanger is $250 \mathrm{~kg}$. Hence modification of new hanger is done with greater productivity.

\section{New hanger design}

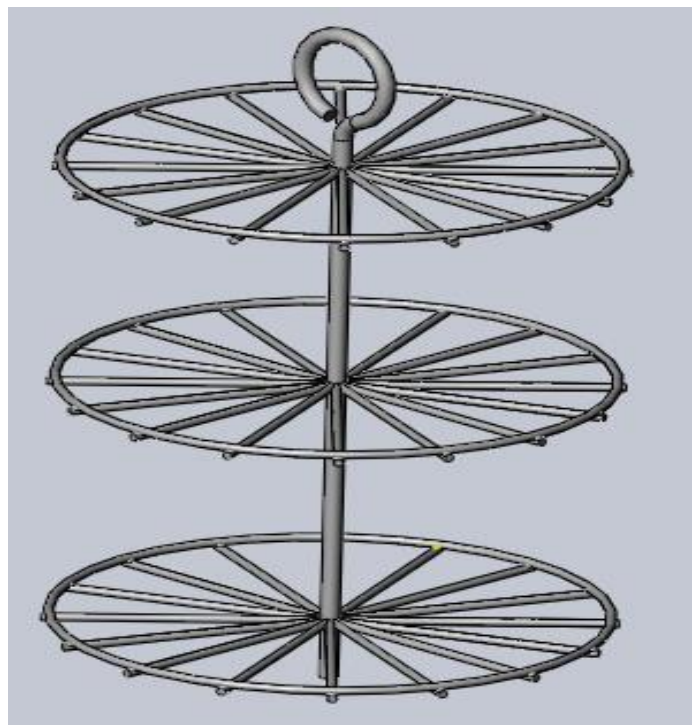

Fig.4. Proposed model of hanger

\section{ANALYSIS OF HANGER}

Table 1. Properties of Structural steel

\begin{tabular}{|l|l|}
\hline Properties & Values \\
\hline Density & $7850 \mathrm{Kg} / \mathrm{m}^{3}$ \\
\hline Young's Modulus & $2 * 10^{5} \mathrm{Mpa}$ \\
\hline Poisson's ratio & 0.3 \\
\hline Bulk Modulus & $1.6667 * 10^{5} \mathrm{Mpa}$ \\
\hline Shear Modulus & $7.6923^{*} 10^{4} \mathrm{Mpa}$ \\
\hline Tensile yield strength & $2.5^{*} 10^{2} \mathrm{Mpa}$ \\
\hline $\begin{array}{l}\text { Tensile ultimate } \\
\text { strength }\end{array}$ & $4.6^{*} 10^{2} \mathrm{Mpa}$ \\
\hline
\end{tabular}

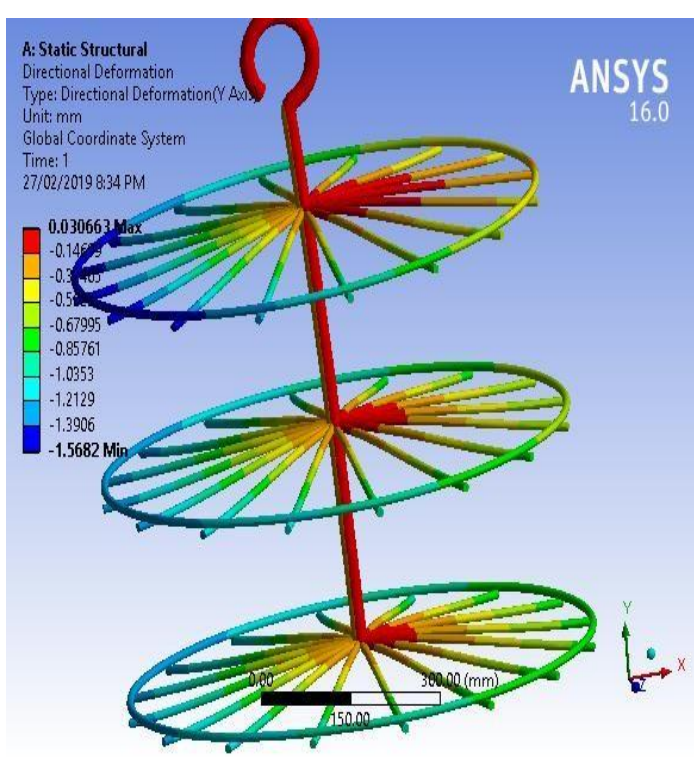

Fig.5. Equivalent stress: 87.64 N/mm (Max.)

\subsection{Ansys Result}

By observing generated equivalent stress value which is less than yield stress of structural steel, hence Design is safe as a principle of Theories of failure.

Deformation due to load is negligible.

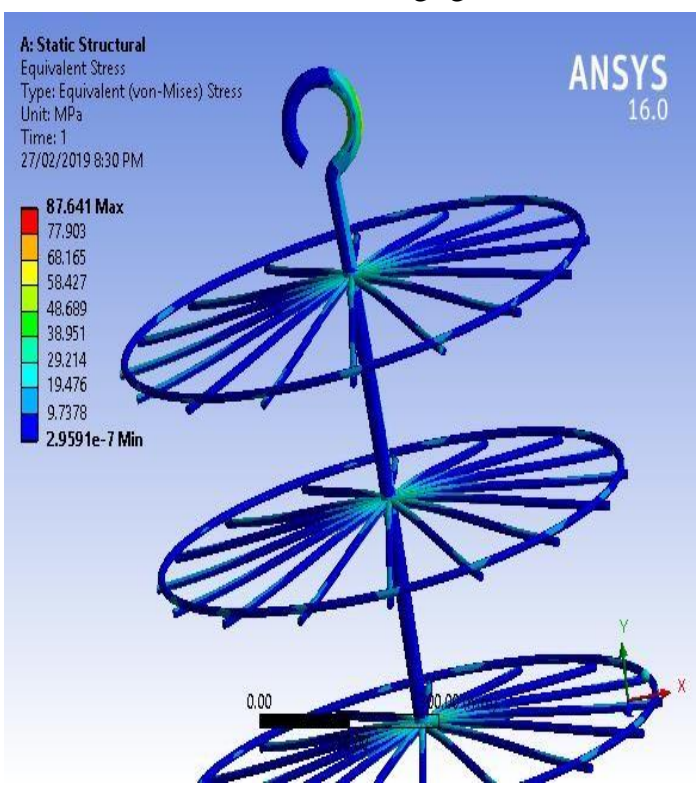

Fig.6. Deformation: 0.03 (Max.) 


\section{EXPERIMENTAL TESTING}

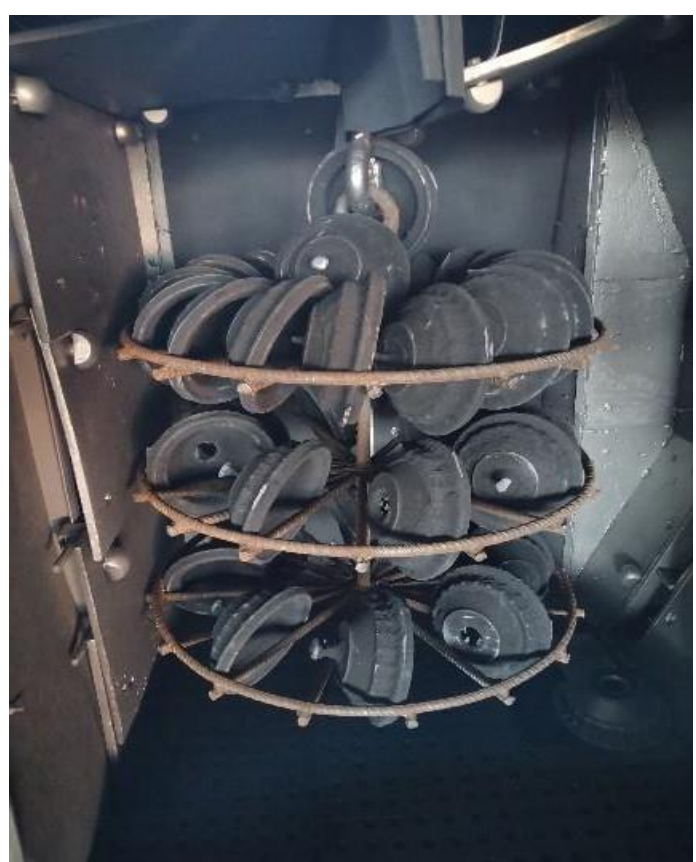

Fig.7. Hanger with Casting before Shot Blasting

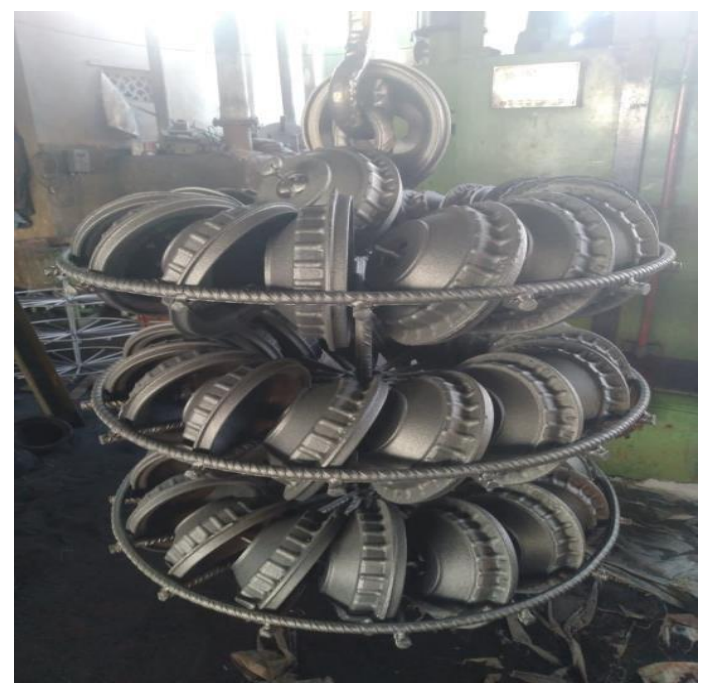

Fig.8.Hangers with Casting after Shot Blasting

\section{CONCLUSION}

In this project work, castings carrying capacity of hanger is increase hence production rate of brake drum increases by using newly design hanger as compare to existing hanger using in shot blasting machine. Following table represent the work conclusion.
Table 2. Comparison of existing and new hanger

\begin{tabular}{|l|c|c|}
\hline Parameters & $\begin{array}{c}\text { Existing } \\
\text { Hanger }\end{array}$ & New Hanger \\
\hline $\begin{array}{l}\text { Number of Casting } \\
\text { produce }\end{array}$ & 16 & 48 \\
\hline No of Cycle & 2 & 1 \\
\hline Total Cycle time & $41 \mathrm{~min}$ & $93 \mathrm{~min}$ \\
\hline $\begin{array}{l}\text { Time required for } \\
\text { producing 1 casting. }\end{array}$ & $2.56 \mathrm{~min}$ & $1.94 \mathrm{~min}$ \\
\hline
\end{tabular}

\section{REFERENCES}

[1] Review on Shot Blasting Processes Mitul Malli M (CAD/CAM) Mechanical Engineering Department, A.D.Patel Institute of Technology NewV.V.Nagar Gujarat India- 300120

[2] Hook Design and Analysis G Bhagyaraj1, K Suryaprakash2, K Subba Rao 3

[3] Analysis of Stress and Deflection of Cantilever Beam and its Validation Using ANSYS Ashis Kumar Samal, T. Eswara Rao.

[4]Methodology of Testing Shot Blasting Machines in Industrial Conditions R. Wrona*,P. Zyzaka, E. Ziółkowskia, M. Brzezinski AGH University of Science and Technology Faculty of Foundry Engineering, ul. Reymont a 23, 30-059, Kraków, Poland a Department of Foundry Processes Engineering, AGH University of Science and Technology, ul.Reymont a 23, 30-059 Kraków, Poland.

[5] New Development in shot blasting machine. Mr. P.A. Patel, Patel Furnace \& Forging Pvt Ltd.

[6] www.pshotblast.com,patel@ satyam.net.in. 